\title{
Parametrization and Validation of Geometry-Based Stochastic Channel Model for Urban Small Cells at $10 \mathrm{GHz}$
}

\author{
Antti Roivainen, Pekka Kyösti, Claudio Ferreira Dias, Veikko Hovinen, \\ Nuutti Tervo, Marko Sonkki, and Matti Latva-aho
}

\begin{abstract}
We derive a full measurement-based parametrization for a 3-D geometry-based stochastic channel model for an urban microcell type of environment at $10 \mathrm{GHz}$. The measurements were performed with a vector network analyzer and dual polarized virtual arrays at the bandwidth of $500 \mathrm{MHz}$. The proposed parametrization is validated by channel simulations. Multiple-input multiple-output channel is reconstructed from the measured data and compared with channel generated by the quasi deterministic radio channel generator using the proposed statistical parameters. The capacity and eigenvalue distribution are used as the validation metrics, showing almost perfect match between the reconstructed and simulated channels.
\end{abstract}

Index Terms-3-D, geometry-based stochastic channel model (GSCM), multiple-input multiple-output (MIMO), polarization, statistical parameters, validation, virtual antenna array.

\section{INTRODUCTION}

In the geometry-based stochastic channel model (GSCM), the propagation channel is characterized by statistical parameters obtained from the radio channel measurements. This gives a possibility to use the same model framework for the simulations at different frequencies and with the different number or type of antennas. Several measurement campaigns have been carried out for investigating the radio channel at the frequency bands above $6 \mathrm{GHz}$. A large number of measurements have been reported for 28, 38, and $60 \mathrm{GHz}$. Several research projects including industry and academia, e.g., METIS [1], 5GCM [2], mmMagic [3], and NYU WIRELESS [4] have been targeting to fulfill the requirements for designing and evaluating new channel models for the frequency bands up to $100 \mathrm{GHz}$.

The lowest frequency band where wide frequency spectrum is not yet licensed for commercial communication systems is around $10 \mathrm{GHz}$. Recently, the parameters have been published based on ray-tracing for an urban microcell (UMi) propagation scenario at $10 \mathrm{GHz}$ in [2]. However, the full parametrization does not exist yet. In [3], a new frequency dependent term has been introduced to model

This research was supported in part by the Finnish Funding Agency for Technology and Innovation (Tekes), in part by Huawei Tech-nologies, in part by Nokia, and in part by Keysight Technologies, Finland. (Corresponding author: Antti Roivainen.)

A. Roivainen was with the Centre for Wireless Communications, University of Oulu, 90014 Oulu, Finland. He is now with Keysight Technologies Finland Oy, 90590 Oulu, Finland (e-mail: antti.roivainen@keysight.com).

P. Kyösti is with the Centre for Wireless Communications, University of Oulu, 90014 Oulu, Finland, and Keysight Technologies Finland Oy, 90590 Oulu, Finland (e-mail: pekka.kyosti@oulu.fi).

C. Ferreira Dias is with the Faculty of Electrical Engineering and Computation, University of Campinas, Campinas 13083-970, Brazil (e-mail: aplnx@decom.fee.unicamp.br).

V. Hovinen, N. Tervo, M. Sonkki, and M. Latva-aho are with the Centre for Wireless Communications, University of Oulu, 90014 Oulu, Finland (e-mail: veikko.hovinen@oulu.fi; nuutti.tervo@oulu.fi;nmarko.sonkki@oulu.fi; matti.latva-aho@oulu.fi). parameters, and the initial parametrization has been determined over the frequency bands 10-80 GHz. However, the specific parametrization has not been given to the $10-\mathrm{GHz}$ frequency band. This motivated us to carry out the radio channel measurements, and to derive full 3-D parametrization for 3rd Generation Partnership Project (3GPP) 3-D type of GSCM at $10 \mathrm{GHz}$. The contributions of this communication are as follows.

1) We present the full 3-D parametrization for GSCM in the microcell type of propagation environment at $10-\mathrm{GHz}$ frequency band.

2) We verify the proposed parametrization by running the channel simulations with quasi deterministic radio channel generator (QuaDRiGa) channel model implementation [5].

The rest of this communication is organized as follows. The radio channel measurements are described in Section II. Section III presents the proposed model parameters, followed by the model validation in Section IV. Finally, the conclusions are drawn in Section V.

\section{Radio Channel Measurements}

The radio channel measurements were carried out with similar setup as in [6] and [7]. Keysight N5247A four-port PNA-X, virtual arrays with dual polarized antenna elements [8], power amplifiers (PAs), and low-noise amplifiers (LNAs) were used. The transmitter $(\mathrm{Tx})$ virtual array was $5 \times 5$ planar array with half wavelength interelement spacing in vertical and horizontal directions. In order to characterize an omnidirectional channel at the receiver ( $\mathrm{Rx})$, the $\mathrm{Rx}$ virtual array was a conformal array shaped as a lateral surface of a cube. Each of four vertical sides had $5 \times 5$ planar arrays with dual polarized antenna elements. Hence, the total number of Rx antenna elements was 100 .

As in [7], the propagation paths were extracted by utilizing estimation of signal parameters by rotational invariance techniques. In [9], we used deterministic METIS map-based model [1], which is a simplified RT tool, to compare the estimated multipath components (MPCs) with respect to modeled MPCs with map-based model. The results showed good match between estimated and modeled MPCs, which indicates sufficient estimation accuracy.

The measurement layouts are illustrated in Fig. 1. The measurements were carried out in two different locations in the campus area of the University of Oulu. Both line-of-sight (LOS) and nonLOS (NLOS) propagation scenarios were measured. In the first measurement location [Fig. 1(a)], the Tx array was located on the staircase and the measurements consisted of two different sites. The first site (Tx1, Rx1 LOS) was an alleyway with vegetation on one side. The building walls consisted of a few windows and the surface material of the walls was a marble slab. At the second measurement site ( $\mathrm{T} \times 2, \mathrm{Rx} 2 \mathrm{LOS}$, and $\mathrm{R} \times 2$ NLOS), the propagation environment was urban street canyon type of environment where there were office buildings with the surface material of concrete on both sides of the street canyon. In the second location, the measurement environment consisted of five-story office buildings with the heights 


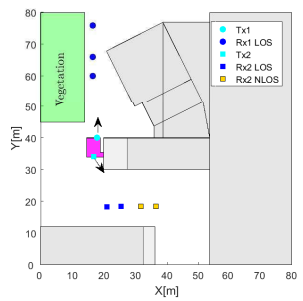

(a)

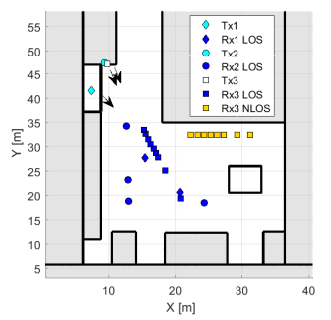

(b)
Fig. 1. Measurement spots and layouts. (a) Location 1. (b) Location 2. Green and gray colors indicate the vegetation and buildings. The same type of Tx and Rx markers designate the measurement from the Tx array to the $\mathrm{Rx}$ array. Black arrows illustrate the boresights of the Tx array.

TABLE I

Main Measurement Settings

\begin{tabular}{|l|c|}
\hline Center frequency & $10.1 \mathrm{GHz}$ \\
\hline Bandwidth & $500 \mathrm{MHz}$ \\
\hline VNA Rx IF bandwidth & $100 \mathrm{kHz}$ \\
\hline VNA output power & $10 \mathrm{dBm}$ \\
\hline RF cable attenuation [Tx, Rx] & $19 \mathrm{~dB}, 10 \mathrm{~dB}$ \\
\hline Amplifier gain [PA, LNA] & $29 \mathrm{~dB}, 25 \mathrm{~dB}$ \\
\hline PA 1 dB compression point & $25 \mathrm{dBm}$ \\
\hline LNA noise figure & $1.8 \mathrm{~dB}$ \\
\hline Transmission power (EIRP) & $23 \mathrm{dBm}$ \\
\hline Measurement dynamic range [LOS, NLOS] & $>40 \mathrm{~dB},>20 \mathrm{~dB}$ \\
\hline Antenna height [Tx, Rx] & $10 \mathrm{~m}, 2 \mathrm{~m}$ \\
\hline Number of frequency points & 401 \\
\hline Total number of measurement spots [LOS, NLOS] & 21,11 \\
\hline
\end{tabular}

of approximately $15 \mathrm{~m}$. The material of the building walls is a mixture of concrete and horizontal corrugated metal siding.

The Tx virtual array positions were selected to represent a possible location for base station and the virtual array was downtilted by $5^{\circ}$ with respect to horizon in all measurements. The lengths of RF-cables were 28 and $10 \mathrm{~m}$ at the $\mathrm{Tx}$ and $\mathrm{Rx}$, respectively. Therefore, the maximum link distance in the measurements was around $36.5 \mathrm{~m}$. Both measurement locations were static during the data recordings. The main measurement settings are given in Table I.

\section{MOdel PARAMETERS}

The model parameters are extracted by following the methodologies given in WINNER channel model documentation [10] and in [7]. Therefore, the parameters are directly applicable to WINNER/QuaDRiGa/3GPP 3-D type of GSCM. In addition, we compare our model parameters with recently published measured parameters in UMi propagation scenario at the frequency band close to $10 \mathrm{GHz}$. In general, there are very limited number of measured model parameters available for this frequency band in the existing literature.

\section{A. Path Loss Models}

Recently, there has been extensive discussion on the path loss (PL) modeling methods. For instance, the prediction accuracy, sensitivity and stability of different PL modeling options for 5G wireless communications have been addressed in [11]. However, the modeling method is still an open issue in $5 \mathrm{G}$ wireless communications. Therefore, we consider two different PL models for both propagation conditions, which are widely used in the literature. These models are the floating intercept (FI) PL model [12] also called alpha-betagamma model and the close-in (CI) free space reference distance PL model [13], where the beta term has been replaced by the free space path loss (FSPL) at $1 \mathrm{~m}$ reference distance.

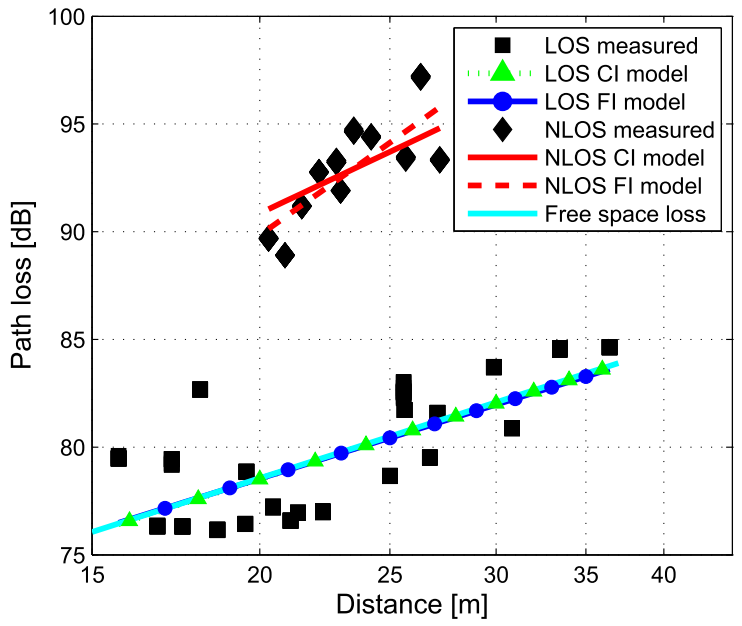

Fig. 2. Path loss in the LOS and NLOS scenarios.

TABLE II

PATH LOSS MODELS

\begin{tabular}{|c|c|c|c|}
\hline & & LOS & NLOS \\
\hline Tx-Rx distance $[\mathrm{m}]$ & $d$ & $15.7-36.5$ & $20-27.5$ \\
\hline \multirow{2}{*}{$P L_{\mathrm{FI}}=\beta+10 \alpha \log _{10}(d)$} & $\alpha$ & 2.0 & 4.4 \\
\cline { 2 - 4 } & $\beta[\mathrm{dB}]$ & 53.2 & 32.2 \\
\hline$P L_{\mathrm{CI}}=\mathrm{FSPL}_{1 \mathrm{~m}}+10 n \log _{10}(d)$ & $n$ & 2.0 & 3.0 \\
\hline
\end{tabular}

Fig. 2 shows the measured basic transmission losses and the modeled PL with both methods in the LOS and NLOS cases. In addition, the free space loss is included as a reference. As clearly observable from Fig. 2, the FI model (dashed red line) gives better match with the measurement data in NLOS scenario. On the other hand, when going to the short link distances the FI model would give too optimistic PL values. This is also seen in [14], where the FI model has been given to LOS scenario at $11 \mathrm{GHz}$. Hence, we can conclude that FI model is more appropriate but must be applied only to the distance range present in Table II. However, in order to cover wider range of link distances we choose the CI PL model. Table II summarizes our PL models at $10.1 \mathrm{GHz}$.

\section{B. Large-Scale Parameters and Cross-Polarization Ratio}

The root mean square delay spread (DS), the shadow fading (SF), the Ricean K-factor (KF), the azimuth angle spread (AS) of departure (ASD), the azimuth AS of arrival (ASA), the elevation AS of departure (ESD), and the elevation AS of arrival (ESA) form the LSPs of the GSCM. All LSPs are log-normally distributed. Fig. 3 shows cumulative distribution function (CDF) for measured LSPs excluding SF. In addition, corresponding simulated curves are added as dashed lines. The simulated curves are produced by QuaDRiGa channel model implementation [5] with the proposed parametrization presented in Tables II-VI.

The mean values of DS are 21.45 and $41.29 \mathrm{~ns}$ for the LOS and NLOS, respectively. In [3], the mean values of 14.1 and $58.9 \mathrm{~ns}$ are reported for LOS and NLOS at $10 \mathrm{GHz}$. Equivalently, the mean values of 8.9 and $46.39 \mathrm{~ns}$ are given at $11 \mathrm{GHz}$ in [15]. In general, the DS depends on the environment and it is sensitive to the dynamic range in the measurements. If the dynamic range is low, e.g., below $20 \mathrm{~dB}$, the MPCs arriving with long delay might vanish to the system noise resulting in a small DS. Our $\sigma_{\mathrm{SF}}$, is small due to shorter variations of link distances and static measurement environment. 


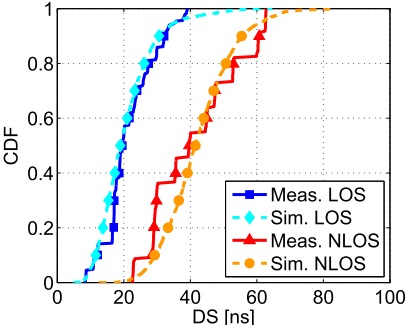

(a)

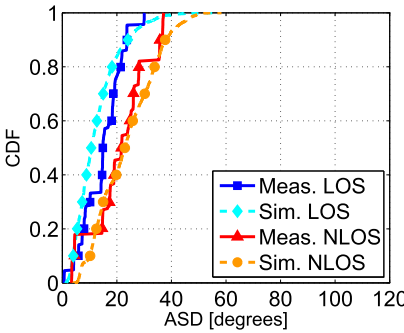

(c)

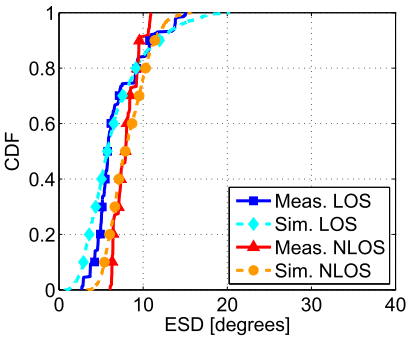

(e)

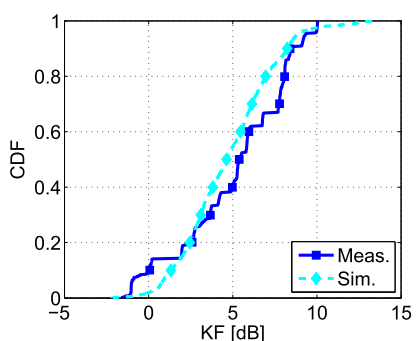

(b)

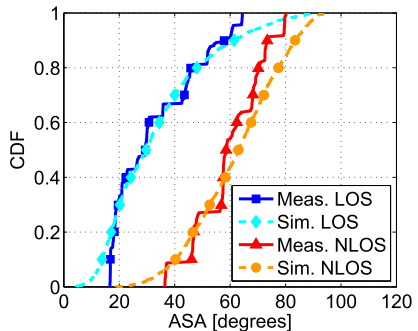

(d)

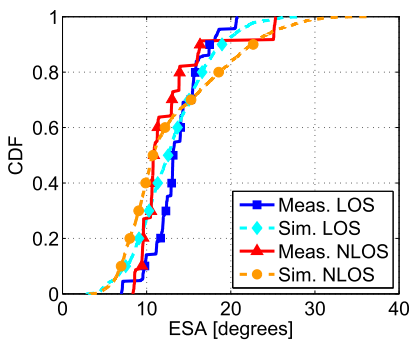

(f)
Fig. 3. CDFs for (a) DS, (b) KF, (c) ASD, (d) ASA, (e) ESD, and (f) ESA.

TABLE III

LSPs, XPR, AND DELAY SCALING PARAMETER

\begin{tabular}{|l|c|c|c|}
\hline \multicolumn{2}{|c}{} & LOS & NLOS \\
\hline \multirow{2}{*}{$\mathrm{DS} \log _{10}([\mathrm{~s}])$} & $\mu_{\mathrm{DS}}$ & -7.70 & -7.41 \\
\cline { 2 - 4 } & $\sigma_{\mathrm{DS}}$ & 0.16 & 0.14 \\
\hline \multirow{2}{*}{$\mathrm{KF}[\mathrm{dB}]$} & $\mu_{\mathrm{KF}}$ & 5.1 & N/A \\
\cline { 2 - 4 } & $\sigma_{\mathrm{KF}}$ & 3.2 & N/A \\
\hline $\mathrm{SF}[\mathrm{dB}]$ & $\sigma_{\mathrm{SF}}$ & 2.0 & 1.5 \\
\hline \multirow{2}{*}{$\mathrm{ASD} \log _{10}\left(\left[^{\circ}\right]\right)$} & $\mu_{\mathrm{ASD}}$ & 1.08 & 1.24 \\
\cline { 2 - 4 } & $\sigma_{\mathrm{ASD}}$ & 0.35 & 0.32 \\
\hline \multirow{2}{*}{$\mathrm{ASA} \log _{10}\left(\left[^{\circ}\right]\right)$} & $\mu_{\mathrm{ASA}}$ & 1.47 & 1.77 \\
\cline { 2 - 4 } & $\sigma_{\mathrm{ASA}}$ & 0.20 & 0.09 \\
\hline \multirow{2}{*}{$\mathrm{ESD} \log _{10}\left(\left[^{\circ}\right]\right)$} & $\mu_{\mathrm{ESD}}$ & 0.80 & 0.89 \\
\cline { 2 - 4 } & $\sigma_{\mathrm{ESD}}$ & 0.17 & 0.07 \\
\hline \multirow{2}{*}{ ESA $\log _{10}\left(\left[^{\circ}\right]\right)$} & $\mu_{\mathrm{ESA}}$ & 1.12 & 1.08 \\
\cline { 2 - 4 } & $\sigma_{\mathrm{ESA}}$ & 0.10 & 0.13 \\
\hline \multirow{2}{*}{ XPR $[\mathrm{dB}]$} & $\mu_{\mathrm{XPR}}$ & 12.4 & 8.2 \\
\cline { 2 - 4 } & $\sigma_{\mathrm{XPR}}$ & 3.8 & 7.2 \\
\hline \multicolumn{2}{|c|}{ Delay scaling parameter $r_{\tau}$} & 3.1 & 1.8 \\
\hline
\end{tabular}

In LOS and NLOS scenarios, the mean values of ASD are $14.89^{\circ}$ and $21.09^{\circ}$, whereas the corresponding mean values of ASA are $32.64^{\circ}$ and $59.69^{\circ}$. In [15], the mean values of ASD are $21.18^{\circ}$ and $27.12^{\circ}$ for LOS and NLOS, whereas the mean ASA of $64.79^{\circ}$ is given for both propagation conditions. In the LOS and NLOS, our mean values for ESD are $6.82^{\circ}$ and $7.96^{\circ}$, whereas the corresponding mean values of ESA are $6.99^{\circ}$ and $13.01^{\circ}$. According to our best knowledge, our elevation ASs are the first elevation AS parameters reported based on measurements for this frequency band and propagation scenario in the existing literature.
TABLE IV

CROSS-CORRELATIONS

\begin{tabular}{|l|c|c|c|c|c|c|c|}
\hline & DS & KF & SF & ASD & ASA & ESD & ESA \\
\hline \multirow{2}{*}{ DS } & 1 & -0.44 & 0.56 & 0.59 & 0.58 & 0.81 & 0.33 \\
& 1 & N/A & -0.21 & 0.82 & -0.35 & 0.41 & -0.42 \\
\hline \multirow{2}{*}{ KF } & -0.44 & 1 & -0.29 & -0.40 & -0.51 & -0.43 & -0.51 \\
& N/A & 1 & N/A & N/A & N/A & N/A & N/A \\
\hline \multirow{2}{*}{ SF } & 0.56 & -0.29 & 1 & -0.04 & 0.70 & 0.67 & -0.04 \\
& -0.21 & N/A & 1 & -0.38 & 0.32 & 0.17 & 0.50 \\
\hline \multirow{2}{*}{ ASD } & 0.59 & -0.40 & -0.04 & 1 & 0 & 0.54 & 0.42 \\
& 0.82 & N/A $^{*}$ & -0.38 & 1 & -0.20 & 0.31 & -0.72 \\
\hline \multirow{2}{*}{ ASA } & 0.58 & -0.51 & 0.70 & 0 & 1 & 0.58 & 0.29 \\
& -0.35 & N/A & 0.32 & -0.2 & 1 & 0.43 & -0.03 \\
\hline \multirow{2}{*}{ ESD } & 0.81 & -0.43 & 0.67 & 0.54 & 0.58 & 1 & 0.36 \\
& 0.41 & N/A & 0.17 & 0.31 & 0.43 & 1 & -0.26 \\
\hline \multirow{2}{*}{ ESA } & 0.33 & -0.51 & -0.04 & 0.42 & 0.29 & 0.36 & 1 \\
& -0.42 & N/A* & 0.50 & -0.72 & -0.03 & -0.26 & 1 \\
\hline \multirow{2}{*}{$* 0$ in the simulations } \\
Upper and lower values in each cell correspond the cross-correlation \\
coefficient in LOS and NLOS scenarios, respectively. \\
\hline
\end{tabular}

TABLE V

CORRELATION DistanCES

\begin{tabular}{|c|c|c|c|}
\hline \multicolumn{2}{|c|}{} & LOS & NLOS \\
\hline \multirow{4}{*}{$\begin{array}{c}\text { Correlation } \\
\text { distance [m] }\end{array}$} & DS & 3 & 3 \\
\cline { 2 - 4 } & ASD & 3 & 3 \\
\cline { 2 - 4 } & ASA & 3 & 3 \\
\cline { 2 - 4 } & SF & 3 & 3 \\
\cline { 2 - 4 } & $\mathrm{KF}$ & 3 & N/A $^{*}$ \\
\cline { 2 - 4 } & ESA & 3 & 3 \\
\cline { 2 - 4 } & ESD & 3 & 3 \\
\hline$* 1 \mathrm{~m}$ in the simulations \\
\hline
\end{tabular}

Cross-polarization ratio (XPR) is first determined for both vertical and horizontal transmission polarizations. The XPRs of vertical and horizontal transmission polarizations are close to each other in the first measurement location where the surfaces of the walls are flat. In the second measurement location, the XPR of vertical transmission polarization $\left(\mathrm{XPR}_{V}\right)$ is around $5 \mathrm{~dB}$ higher than the XPR of horizontal transmission polarization $\left(\mathrm{XPR}_{H}\right)$ in both propagation conditions. Although the magnitude of reflection coefficient at perpendicular incidence is typically larger than the magnitude of parallel reflection coefficient [16], the different polarization components are also heavily dependent on the roughness and material of the surfaces of surrounding buildings. Therefore, the physical explanation for the difference between $\mathrm{XPR}_{V}$ and $\mathrm{XPR}_{H}$ in the second measurement location is the roughness of wall surfaces, i.e., horizontal corrugated metal siding that causes larger channel depolarization and thus smaller XPR for horizontal transmission polarization.

The XPR of the model is determined as the mean value over all $\mathrm{XPR}$ results, i.e., $\mathrm{XPR}_{V}$ and $\mathrm{XPR}_{H}$. Table III summarizes the LSPs, $\mathrm{XPR}$, and delay scaling parameter of the model.

\section{Correlation Parameters}

In the model, the LSPs have to be generated with correlation properties [12]. The cross-correlations are modeled by cross correlation coefficients between all pairs of LSPs. The cross-correlation coefficients are shown in Table V. Some LSPs have strong dependence of each other, for instance, ESD \& DS and ASD \& DS in the LOS and NLOS scenarios, respectively. Equivalently, some LSPs do not have any correlation or the correlation is very small, e.g., ASD \& ASA and ESA \& SF in the LOS case.

Correlation distances are calculated from the subset of our measurements. More precise, the correlation distances are determined 


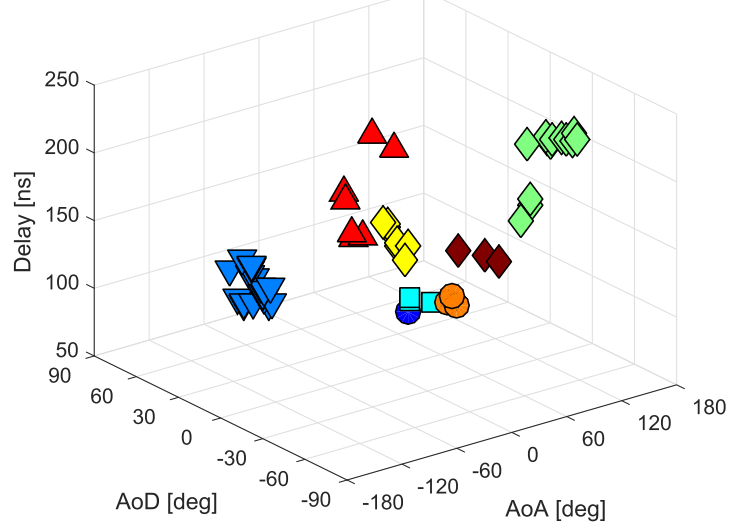

(a)

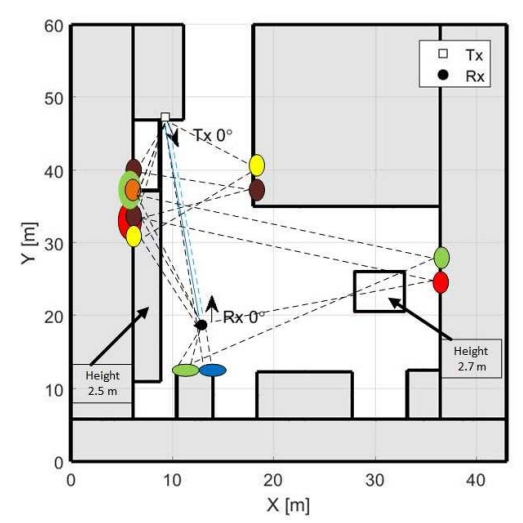

(b)

Fig. 4. LOS scenario, an example of (a) identified clusters and (b) physical locations of clusters.

based on the LSP auto-correlation functions estimated from the data of closely located Rx positions at the second measurement location marked as squares in Fig. 1(b). These measurements were performed with approximately 1-m increment of link distances. The correlation distances are presented in Table V. Correlation distances are the same in both scenarios $(3 \mathrm{~m})$. However, it should be noted that based on our measurements we can only approximate the correlation distance. Moreover, the amount of measurement points and the range of link distances are somewhat limited for accurate correlation distance analysis. In order to determine more accurate correlation distances, a substantially larger amount of measurement data with the continuous data recording over mobile movement would have been needed.

\section{Cluster Parameters}

We use KPowerMeans [17] algorithm for grouping propagation paths to the clusters. MC distance (MCD) [18] including powers of propagation paths is used as a metric for calculating the multidimensional distances between propagation paths and cluster centroids. Propagation paths are set to the clusters based on the shortest MCD. Several validation indices have been studied and proposed for determining the optimal number of clusters in the open literature. We choose the optimal solution for the number of clusters based on the combination of Caliñski-Harabasz and Davies-Bouldin validation indices [17].

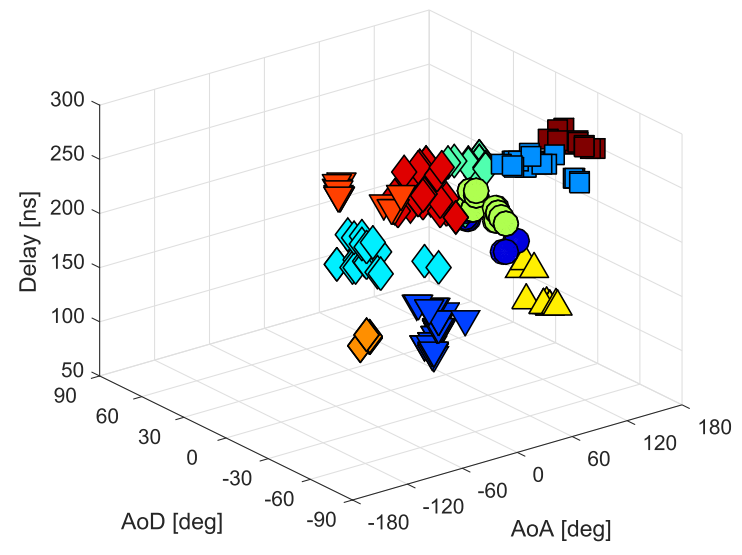

(a)

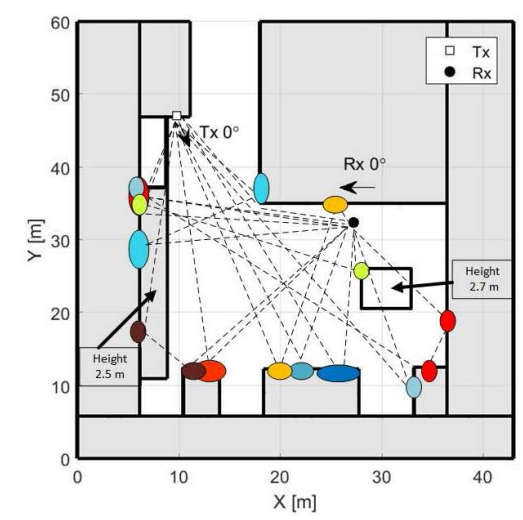

(b)

Fig. 5. NLOS scenario, an example of (a) identified clusters and (b) physical locations of clusters.

TABLE VI

Cluster Parameters

\begin{tabular}{|l|c|c|}
\hline & LOS & NLOS \\
\hline Number of clusters $^{\circ}$ & 8 & 10 \\
\hline Cluster ASD [ $^{\circ}$ & 3 & 7 \\
\hline Cluster ASA [ $^{\circ}$ ] & 5 & 8 \\
\hline Cluster ESD $^{\circ}$ ] & 3 & 5 \\
\hline Cluster ESA [ $^{\circ}$ ] & 3 & 7 \\
\hline Per cluster shadowing std $\zeta[\mathrm{dB}]^{-}$ & 6 & 3 \\
\hline
\end{tabular}

The examples of identified clusters and the illustration of their physical locations based on angles and delays in the LOS and NLOS measurements are displayed in Figs. 4 and 5. In addition to direct LOS path and the cluster consisting of ground reflected paths, two single-bounce clusters and four multibounce clusters (either double- or triple-bounce clusters) are observed in the LOS example. The gains of clusters decrease as the delays of MPCs and the number of bounces increase. In the NLOS case, the strongest cluster is composed of a single-bounce specular reflection.

Table VI summarizes cluster parameters of the model. The number of clusters and the cluster ASs are smaller in comparison to corresponding parameters determined at the lower frequency bands, e.g., in 3GPP 3-D channel model [19]. The possible physical explanation for smaller cluster ASs in comparison to lower frequency bands is that the specular reflection is more dominant propagation mechanism in comparison to diffuse scattering. Similar observations have been presented, e.g., in [20]. 


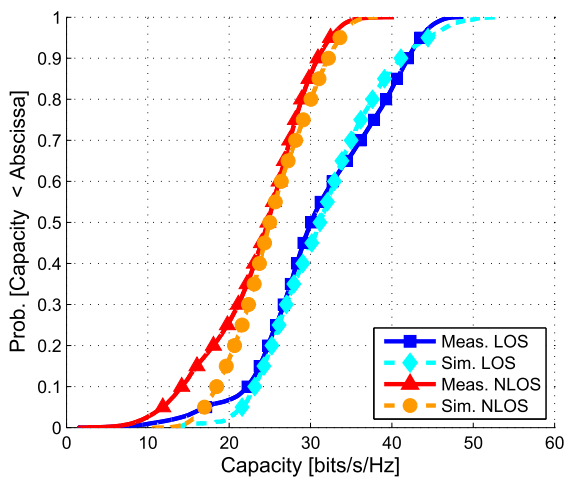

(a)

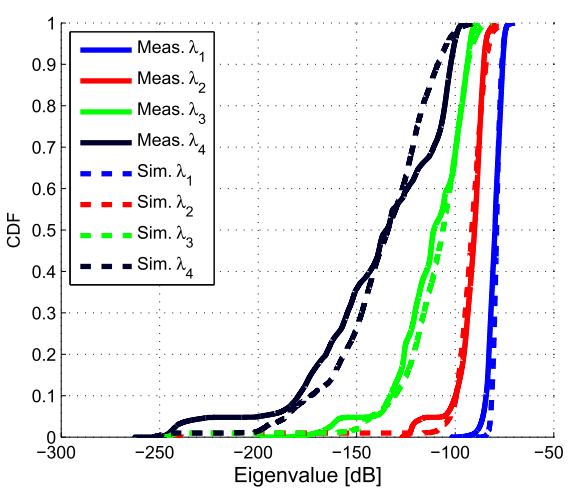

(b)

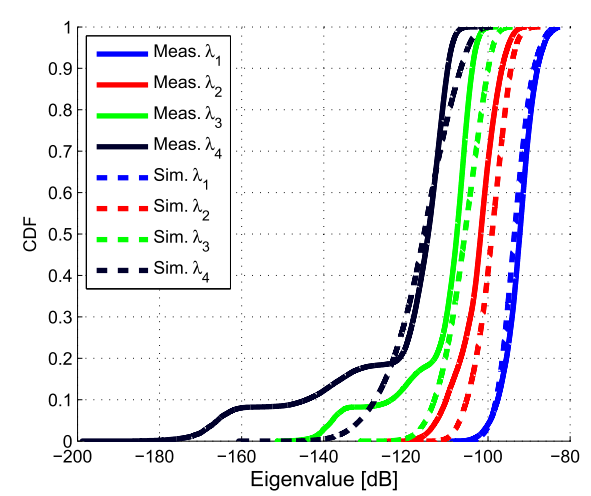

(c)

Fig. 6. Validation results of a) channel capacity, b) eigenvalues in LOS, and c) eigenvalues in NLOS.

\section{Model VALidation}

The proposed parameters are validated by channel simulations. Radio channel is directly reconstructed by embedding antennas to the propagation path estimates, and equivalently the channel is generated by QuaDRiGa channel model implementation [5] using the proposed model parameters of Tables II-VI. In both cases, the horizontally aligned uniform linear arrays consisting of eight and four vertical polarized ideal dipoles are used as the Tx and Rx antennas, respectively. The spacing between antenna elements is set to half wavelength. Downlink radio channel simulations are performed for single radio link at $10.1-\mathrm{GHz}$ center frequency over $500-\mathrm{MHz}$ bandwidth. The range of link distances and the heights of antennas are the same as in the measurements.

\section{A. Validation Metrics}

In the validation, 30 and $20 \mathrm{~dB}$ dynamic ranges are used for the reconstructed and simulated channels in the LOS and NLOS scenarios, respectively. The reconstruction of channel coefficients from the propagation path estimates is presented in [7]. Commonly used channel capacity is selected as the first validation metric. The eigenvalue distribution validating the spatial structure of the radio channel is selected as the second validation metric. We assume that channel state information at the $\mathrm{Tx}$ is not available. Hence, equal power is allocated among the $\mathrm{Tx}$ antennas and subcarriers. Frequency domain channel for $N_{r} \mathrm{Rx}$ antennas, $N_{t}$ Tx antennas, and $N_{f}$ subcarriers is denoted as $\mathbf{H}_{F} \in \mathbb{C}^{N_{r} \times N_{t} \times N_{f}}$. The squared singular values are equal to the eigenvalues of the channel covariance matrix [21]

$$
\lambda_{i, n_{f}}=\operatorname{eig}\left\{\mathbf{H}_{F} \mathbf{H}_{F}^{H}\right\}
$$

where $\operatorname{eig}\{\cdot\}$ denotes eigenvalue decomposition and $\lambda_{i}$, $n=1, \ldots, n_{\min }, n_{\min }=\min \left(N_{r}, N_{t}\right)$ are the eigenvalues representing the path gains of the spatio-polarimetric subchannels. We calculate the instantaneous capacity for each frequency bin as [21]

$$
C\left(n_{f}, t\right)=\sum_{i=1}^{n_{\min }} \log _{2}\left(1+\frac{P_{T}}{N_{T} N_{0}} \lambda_{i, n_{f}}\right)
$$

where $P_{T}(1 \mathrm{~W})$ is the transmitted power and $N_{0}$ is the thermal noise power determined as

$$
N_{0}=k T B
$$

where $k$ is Boltzmann's constant, $T=290 \mathrm{~K}$ is the noise temperature, and $B(500 \mathrm{MHz})$ is the bandwidth. Alternatively, the signal-to-noise ratio (SNR) could be fixed to constant $\mathrm{dB}$ value in the capacity analysis, like in [7]. However, we use fixed $P_{T}$ leading to varying SNR directly determined by the channel realizations. By doing this, the impact of proposed PL and SF models are also validated.

\section{B. Validation Results}

Fig. 6(a) shows CDFs for measured and simulated capacities. In both scenarios, the measured and simulated capacities are almost equal. The capacity is larger in the LOS scenario than in the NLOS scenario due to higher SNR in LOS condition caused by the smaller PL. However, the capacities between different propagation scenarios are not directly comparable because the range of simulated link distance is not the same.

The measured and simulated eigenvalues in the LOS and NLOS scenarios are displayed in Fig. 6(b) and (c). In the LOS case, the simulated eigenvalues match the measured eigenvalues. The difference between the first and the second eigenvalues is around $10 \mathrm{~dB}$ whereas the difference between the second and third eigenvalues is around $15 \mathrm{~dB}$. The shapes of the first and second eigenvalue curves are vertically stiff indicating that at least two spatial subchannels can be used in the LOS case. The shapes of the third and fourth eigenvalue curves are more gradual. This means that there is more uncertainty for using the third and fourth spatial subchannels in the data transmission. Furthermore, the strength of fourth spatial subchannel might be already too low for practical usage. A small mismatch, around 1-2 dB, can be seen between simulated and measured eigenvalues in the NLOS case. The median values of all eigenvalues are above $-116 \mathrm{~dB}$ which indicates the possibility to use all spatiopolarimetric subchannels for transmission. However, the usability of the weakest spatial subchannels depends on several things, e.g., transmission power and Rx sensitivity.

\section{CONCLUSION}

We presented the full parametrization for 3-D GSCM in urban small cells at $10-\mathrm{GHz}$ frequency band. The PL exponents of 2.0 and 3.0 were observed for LOS and NLOS propagation scenarios. Due to static measurement environment, the $\sigma_{\mathrm{SF}}$ was 2 and $1.5 \mathrm{~dB}$ for LOS and NLOS propagation conditions. The mean values for ASD, ASA, ESD, and ESA were $14.89^{\circ}, 32.64^{\circ}, 6.82^{\circ}$, and $13.62^{\circ}$ in LOS case. Corresponding parameters for NLOS were $21.09^{\circ}, 59.69^{\circ}, 7.96^{\circ}$, and $12.67^{\circ}$. The number of clusters and the cluster ASs were smaller in comparison to corresponding parameters at lower frequency bands.

The downlink channel simulations were run in order to validate the proposed parametrization in urban small cells at $10.1 \mathrm{GHz}$. 
The channel capacity and eigenvalue distribution were used as the validation metrics. The validation results showed almost perfect match between simulated and reconstructed channels.

\section{ACKNOWLEDGMENT}

The authors would like to thank M. Wirtanen for assisting in the measurements.

\section{REFERENCES}

[1] L. Raschkowski, P. Kyösti, K. Kusume, and T. Jämsä, Eds., "METIS channel models, deliverable D1.4 V3," Tech. Rep. ICT-317669 METIS Project, 2015. [Online]. Available: https://www.metis2020. com/documents/deliverables/

[2] Aalto University, BUPT, CMCC, Nokia, NTT DOCOMO, New York University, Ericsson, Qualcomm, Huawei, Samsung, INTEL, University of Bristol, KT Corporation, and University of Southern California, "5G channel model for bands up to $100 \mathrm{GHz}$, version 2.3," in Proc. 2nd Workshop Mobile Commun. Higher Freq. Bands (MCHFB) Globelcom, Dec. 2015, pp. 1-102.

[3] "Measurement campaigns and initial channel models for preferred suitable frequency ranges, deliverable D2.1," Millimetre Wave Based Mobile Radio Access Network for Fifth Generation Integrated Communications (mmMAGIC), Tech. Rep. H2020-ICT-671650, 2016. [Online]. Available: https://bscw.5g-mmmagic.eu/pub/bscw.cgi/ d94832/mmMAGIC_D2-1.pdf

[4] NYUSIM: The Open Source 5G Channel Model Simulator Software, accessed on Jun. 22, 2016. [Online]. Available: http://wireless. engineering.nyu.edu/5g-millimeter-wave-channel-modeling-software/

[5] Fraunhofer Heinrich Hertz Institute. (2016). The Implementation of Quasi Deterministic Radio Channel Generator (QuaDRiGa), v1.4.1-551. [Online]. Available: http://www.quadriga-channel-model.de

[6] C. Ferreira Dias et al., "Spatial radio channel sounding for static environment at $10 \mathrm{GHz}$," in Proc. IEEE Eur. Conf. Antennas Propag., Davos, Switzerland, Apr. 2016, pp. 1-5.

[7] A. Roivainen, C. Ferreira Dias, N. Tervo, V. Hovinen, M. Sonkki, and M. Latva-Aho, "Geometry-based stochastic channel model for two-story lobby environment at $10 \mathrm{GHz}$," IEEE Trans. Antennas Propag., vol. 64, no. 9, pp. 3990-4003, Sep. 2016

[8] M. Sonkki, V. Hovinen, N. Tervo, C. Ferreira Dias, J. Meinilä, and A. Roivainen, "Dual-polarized patch antenna for virtual antenna array based radio channel measurements at $10 \mathrm{GHz}$," in Proc. IEEE Eur. Conf. Antennas Propag., Lisbon, Portugal, Apr. 2015, pp. 1-4.
[9] A. Roivainen, P. Kyoesti, V. Hovinen, and C. Ferreira Dias, "Validation of deterministic radio channel model by $10 \mathrm{GHz}$ microcell measurements," in Proc. Eur. Wireless (EuWI), Workshop Adv. PHY MAC Layer Design $5 G$ Mobile Networks Internet of Things, Oulu, Finland, May 2016, pp. 288-293.

[10] P. Kyösti, "WINNER II channel models, deliverable D1.1.2 V1.0: Part II: Radio channel measurement and analysis results," Tech. Rep. IST-4-027756 WINNER II Project, 2007. [Online]. Available: http://projects.celtic-initiative.org/winner+/deliverables.html

[11] S. Sun et al., "Investigation of prediction accuracy, sensitivity, and parameter stability of large-scale propagation path loss models for $5 \mathrm{G}$ wireless communications," IEEE Trans. Veh. Technol., vol. 65, no. 5, pp. 2843-2860, May 2016.

[12] P. Kyösti, "WINNER II channel models, deliverable D1.1.2 V1.2, part I: Channel models," Tech. Rep. IST-4-027756 WINNER II Project, 2008. [Online]. Available: http://projects.celticinitiative.org/winner+/deliverables.html

[13] T. S. Rappaport, R. W. Heath, Jr., R. C. Daniels, and J. N. Murdock, Millimeter Wave Wireless Communications. Englewood Cliffs, NJ, USA: Prentice-Hall, 2015.

[14] M. Kim, J.-I. Takada, Y. Chang, J. Shen, and Y. Oda, "Large scale characteristics of urban cellular wideband channels at $11 \mathrm{GHz}$," in Proc. IEEE Eur. Conf. Antennas Propag., Lisbon, Portugal, Apr. 2015, pp. 1-4.

[15] J.-I. Takada, M. Kim, and K. Saito, "Characterization of radio propagation channel at $11 \mathrm{GHz}$," in Proc. Eur. Wireless Conf., Oulu, Finland, May 2016, pp. 1-6.

[16] O. Landron, M. J. Feuerstein, and T. S. Rappaport, "A comparison of theoretical and empirical reflection coefficients for typical exterior wall surfaces in a mobile radio environment," IEEE Trans. Antennas Propag., vol. 44, no. 3, pp. 341-351, Mar. 1996.

[17] N. Czink, P. Cera, J. Salo, E. Bonek, J.-P. Nuutinen, and J. Ylitalo, "A framework for automatic clustering of parametric MIMO channel data including path powers," in Proc. IEEE Veh. Technol. Conf., Montreal, QC, Canada, Sep. 2006, pp. 1-5.

[18] N. Czink, P. Cera, J. Salo, E. Bonek, J.-P. Nuutinen, and J. Ylitalo, "Improving clustering performance using multipath component distance," Electron. Lett., vol. 42, no. 1, pp. 33-35, Jan. 2006.

[19] 3rd Generation Partnership Project, "Study on 3D channel model for LTE TR 36.873, v12.2.0," Tech. Rep. V. 12.2.0., 2015.

[20] W. Fan, I. Carton, J. Ø. Nielsen, K. Olesen, and G. F. Pedersen, "Measured wideband characteristics of indoor channels at centimetric and millimetric bands," EURASIP J. Wireless Commun. Netw., vol. 58 , pp. 1-13, Feb. 2016.

[21] D. Tse and P. Viswanath, Fundamentals of Wireless Communication. Cambridge, U.K.: Cambridge Univ. Press, 2005. 